\title{
Synthetic single crystal diamonds for X-ray optics
}

Shevyrtalov, Sergey, Barannikov, Alexander, Palyanov, Yurii, Khokhryakov, Alexander, Borzdov , Yuril, et al.

SPIE. Event: SPIE Optics + Optoelectronics, 2021, Online Only 


\title{
Synthetic single crystal diamonds for X-ray optics
}

\author{
Sergey Shevyrtalov*a, Alexander Barannikov"a ${ }^{\text {Y }}$ urii Palyanov $^{\text {b,c }}$, Alexander Khokhryakov ${ }^{\text {b,c }}$, \\ Yurii Borzdov $^{\text {b,c }}$, Ilya Sergueev ${ }^{d}$, Sergey Rashchenko ${ }^{c}$ and Anatoly Snigirev ${ }^{\mathrm{a}}$ \\ ${ }^{a}$ Immanuel Kant Baltic Federal University, 236041, Kaliningrad, Russia; ${ }^{\mathrm{b}}$ Sobolev Institute of \\ Geology and Mineralogy, Siberian Branch of the Russian Academy of Sciences, 630090, \\ Novosibirsk, Russia; ${ }^{\mathrm{c}}$ Novosibirsk State University, 630090, Novosibirsk, Russia; ${ }^{\mathrm{d}}$ Deutsches \\ Elektronen-Synchrotron, 22607 Hamburg, Germany
}

\begin{abstract}
In the manuscript we report on characterization of single-crystalline (111) plates prepared from type Ib diamonds with nitrogen content of 100-150 ppm and (100) plates prepared from IIa diamond by means of high-resolution rocking curve imaging (RCI). Contrary to a common opinion about intrinsic poor diffraction quality of type Ib diamonds, RCI showed the presence of nearly defect-free areas of several $\mathrm{mm}^{2}$ in the central part of the (111)-oriented diamond plates. In comparison with the (100)-oriented IIa diamond plates prepared by the same HPHT setup the Ib diamonds possess better diffraction quality. The observed broadening of the rocking curves associates with the cutting and polishing processes, causing strains around the edges of the plates and rare defects. An improvement of preparation technique will thus allow to make single-crystalline diamond plates for Laue and Bragg monochromators and beam splitters from type Ib diamond with areas large enough to be used as wavefront-preserving optical elements at $4^{\text {th }}$ generation synchrotron facilities.
\end{abstract}

Keywords: X-ray optics; single crystal diamond; Bragg beamsplitters; rocking curve imaging

\section{INTRODUCTION}

The emerging new generation of 'diffraction-limited' $4^{\text {th }}$ generation storage rings based on MBA lattice design offers users photon beams of extreme brightness, essential for novel applications that require high coherent flux or nanosized beams [1]. On the other hand, preservation of photon beam phase space and coherent properties becomes an issue, that generally cannot be addressed by X-ray optics routinely used at $3^{\text {rd }}$ generation facilities. Nowadays Si-based optics elements are widespread at the $\mathrm{x}$-ray sources due to the well-established methods of crystal growth and processing, leading to large crystals with a high degree of crystal perfection. However, considering material properties, the preferred material for $\mathrm{x}$-ray optics would be diamond with lower X-ray absorption, lower thermal expansion, and much better thermal conductivity. The benefits of using diamond for beam monochromatization at $4^{\text {th }}$ generation sources include:

1. Better wavefront preservation by diamond monochromators due to smaller thermal 'bumping' compared with silicon devices (a combined effect of low thermal expansion and high thermal conductivity).

2. Better angular stability of water-cooled diamond monochromators compared with cryocooled silicon devices [2], essential for preservation of effective source size.

3. Small X-ray absorption compared to silicon, allowing for implementation of the beam multiplexing schemes [3].

In this context it won't be superfluous to mention that diamond already prove its efficiency as materials of choice for compound refractive lenses (CRLs). Diamond lenses were introduced in both planar and rotational parabolic shapes [4,5] and, recently, as a micro-lenses with rotational parabolic shapes fabricated by focused ion-beam lithography [6]. They were made of single-crystal diamond, which is considered as an ideal material because it does not cause any undesired diffuse scattering, and at the same time withstands high heat-load induced by synchrotron radiation. Moreover, diamond beamsplitters would give much impact on high repetition rate X-ray free electron lasers (XFELs) facilities with providing beam sharing, in-situ beam monitoring and outcoupling in cavity-based XFEL schemes $[7,8]$

EUV and X-ray Optics, Sources, and Instrumentation, edited by René Hudec, Ladislav Pina, Thomas Tschentscher,

Libor Juha, Luc Patthey, Kai Tiedtke, Marco Zangrando, Saša Bajt, Stéphane Guizard, Proc. of SPIE Vol. 11776,

117760G · @ 2021 SPIE · CCC code: 0277-786X/21/\$21· doi: 10.1117/12.2589702 
Diamond crystal plates with (111) surface orientation are of primary importance for diffracting X-ray optics owing to a large intrinsic energy bandwidth of the $\{111\}$ Bragg reflection $\left(\Delta E / E=5.7 \square 10^{-5}\right)$ and the resulting flux of the reflected Xrays compared with those of silicon $[9,10]$. It is worth noting that at $3^{\text {rd }}$ generation facilities, the Laue geometry became standard for diamond monochromators in order to minimize beam footprint on crystal made of material with inhomogeneous lattice quality. A drawback of such a choice is an inevitable degradation of beam phase space because of intrinsic dispersion of Laue geometry. The corresponding decrease in coherent flux and increase in effective source size is negligible in comparison with effects from electron beam emittance and can be tolerated at $3^{\text {rd }}$ generation facilities. However, this is definitely not the case for 4th generation storage rings and X-ray free-electron lasers, where Bragg geometry has to be chosen for coherence-preserving crystal monochromators and beam splitters.

The progress related to the growth of high-quality diamond crystals with at least $2 \mathrm{~mm}^{2}$ defect- and strain-free surface area is very slow. Large diamond single crystals can be grown by high-pressure and high-temperature (HPHT) technique with belt, cubic or split-sphere type apparatuses [11-13]. Depending on nitrogen concentration, diamonds are divided into nitrogen-containing type I, and nitrogen-free type II, containing less than $1 \mathrm{ppm}$ of nitrogen. Type I diamonds are further subdivided into type $\mathrm{Ib}$, containing nitrogen impurity in the form of single substitutional atoms (C-centers), and type Ia, containing aggregated nitrogen forms (A- and B-centers) [14].

The monochromators and the beam-splitters demand for the diamond crystals without dislocations, strains, and significant rocking curve broadening [15]. According to these requirements diamond Bragg (111) and (001) plates with defect-free areas of the order of $5 \mathrm{~mm}^{2}$ were successfully synthesized and tested in the past decade at LCLS and APS $[16,17]$. These plates were manufactured from synthetic diamonds of type IIa (nitrogen-free), previously considered to be the only type suitable for growing crystals with large and homogeneous defect-free areas [18]. However, there are only few papers related to the examination of diffraction quality of nitrogen-containing Ia-b crystals [19,20]. Macrander et al. showed the strain distribution inside the Ib diamond crystal by measuring rocking curve shift across the surface [19]. The shift varied up to $6 \operatorname{arcsec}(29 \mu \mathrm{rad})$ could be related to the non-uniform distribution of nitrogen in the crystal. Hoszowska et al. performed Rocking curve imaging (RCI) of (111)-oriented diamond plates doped with nitrogen and observe $1.5 \operatorname{arcsec}(7.3 \mu \mathrm{rad})$ spread around the crystal attributed to the nitrogen impurities [20]. However, the origin of the lattice imperfection was not clear.

In this work we report the characterization of diffraction quality of $250-\mu \mathrm{m}$ thick type $\mathrm{Ib}$ and IIa diamond plates with (111) and (100) orientation, respectively, cut from HPHT grown single crystals, previously characterized by white beam topography and selective etching method [21]. It was found that dislocation-free volume achieves $58 \mathrm{~mm}^{3}$ and (111)oriented plates cut from the crystal have strain-free area of $25 \mathrm{~mm}^{2}$. Here, we used $\mathrm{x}$-ray high energy resolution $(\Delta \mathrm{E} / \mathrm{E}=$ $10^{-7}$ ) RCI from (333) and (400) reflections in order to obtain detailed information about diamond crystals quality. Two measured plates with $3 \mathrm{~mm}^{2}$ sizes possess defect-free area of $1 \times 2.2 \mathrm{~mm}$ and $1 \times 1.6 \mathrm{~mm}$ with a rocking curve broadening not exceeding $0.6 \mu \mathrm{rad}$ and one (100) plate with $2 \mathrm{~mm}^{2}$ possess a defect-free area of $1.0 \times 1.5 \mathrm{~mm}$ with a RC broadening of $0.23 \mu \mathrm{rad}$.

\section{EXPERIMENTAL DETAILS}

Synthetic diamond crystals were grown by the temperature gradient method using a high-pressure apparatus of the "split sphere" type (BARS) and characterized by white-beam topography, selective etching methods, and IR absorption spectroscopy [21]. The crystals were grown at $P=5.7 \mathrm{GPa}$ and $T=1550{ }^{\circ} \mathrm{C}$ with the use of a $\mathrm{Ni}_{0.7} \mathrm{Fe}_{0.3}$ alloy as a solventcatalyst. The crystallization scheme was described in detail previously [21,22]. Grown diamonds have octahedral shape and brownish-yellow color. An average growth rate was estimated as $39-45 \mu \mathrm{m} / \mathrm{h}$ for the upper $\{111\}$ faces of $2.5-3.0$ carats crystals.

Two (111)-oriented diamond plates with $3 \mathrm{~mm}^{2}$ surface area were cleaved from the outer parts of two single crystals with a slight deviation from the (111) plane. The (100)-oriented plate was mechanically cut and polished from both sides. Surface cleanliness was achieved empirically by combining contact pressure and polishing directions. Characterization of the plates was performed by means of optical microscopy and infrared (IR) absorption spectroscopy. Birefringence 
images were taken using a binocular with a polarization attachment. The IR spectra were measured with a Bruker Vertex 70 FTIR spectrometer using a circular aperture of approximately $3 \mathrm{~mm}$ in diameter. The type and concentrations of nitrogen defects in the diamond plates were determined from the infrared spectra using standard procedures [23]. It was found that the plates contained nitrogen impurities predominantly in the form of isolated substitutional atoms (C-centers, type Ib) with concentrations in the range of $100-150 \mathrm{ppm}$.

A preliminary characterization of the (111) oriented diamond plates was made by Bragg-topography with polychromatic $\mathrm{x}$-ray beam provided by the liquid Ga point laboratory source and by polarization optical microscopy. The high resolution study of the diamond plates quality was carried out by measuring RCI at the PETRA III P01 beamline of the Deutsches Elektronen-Synchrotron (DESY) [24]. The samples were studied at room temperature in the Bragg geometry using monochromatic collimated beam at energy of $14.41 \mathrm{keV}$. The setup includes high-resolution Si (4 00 0)(10 64 ) monochromator provided $\Delta E / E=10^{-7}$ and asymmetric $\operatorname{Si}\left(\begin{array}{lll}4 & 2 & 2\end{array}\right)$ reflection which works as the collimator with asymmetry parameter $b=-0.1$. The beam impinging on the studied diamond plates has energy bandwidth of $1.5 \mathrm{meV}$, angular divergence of 0.6-0.8 $\mu \mathrm{rad}$ and spot size (FWHM) of $11 \times 2 \mathrm{~mm}^{2}$ along and perpendicular to the reflection plane, respectively. Rocking curve imaging were performed by the angular scan of the studied crystals with a step size of 0.17 $\mu \mathrm{rad}$ and by collection data using 2D photon counting Lambda detector (X-Spectrum) with $55-\mu \mathrm{m}$ pixel size and 256x256 pixels total area. The experimental setup is showed in Figure 1. The rocking curves were analyzed for each pixel and the maps of the FWHM and center of mass (COM) of reflection were obtained. The setup was tested using good quality $\operatorname{Si}(555)$ reflection with theoretical width of reflection of $1.46 \mu \mathrm{rad}$. The mean reflection FWHM obtained for all pixels was $1.60 \mu \mathrm{rad}$ giving incoming beam divergence of $0.65 \mu \mathrm{rad}$. The integral reflection FWHM was 1.70 $\mu \mathrm{rad}$ indicating $\sim 0.4 \mu \mathrm{rad}$ spread of the beam direction over entire spot size.

For the RCI we have chosen high order (333) reflection of diamond because of (1) the beam incidence angle, which is $\sim 45$ degrees to the sample, whereas Bragg angle for (333) plane is $38.84^{\circ}$, so that mounting system, which can introduce strains to the plate, is not required, and (2) high sensitivity to small lattice distortions due to the narrow rocking curve with theoretical width of $2.13 \mu \mathrm{rad}(0.46 \mathrm{arcsec})$.

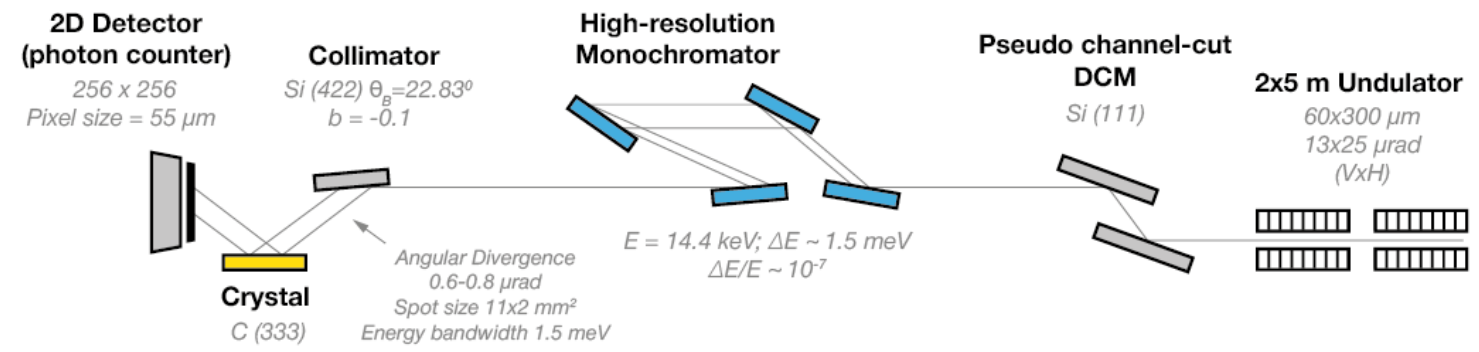

Figure 1. Setup for high-resolution RCI of the diamond (333) reflection at an energy E $=14.41 \mathrm{keV}$. It includes two $5 \mathrm{~m}$ undulators, Si pseudo channel-cut double-crystal monochromator, four-crystal high-resolution $\operatorname{Si}(400)(1064)$ monochromator, asymmetrically cut silicon (422) collimator, the sample and the 2D detector.

\section{RESULTS AND DISCUSSION}

Preliminary analysis of the diamond plates quality has been made using optical polarization microscopy (birefringence images) and Bragg topography on a polychromatic $G a K \alpha(9.2 \mathrm{keV})$ laboratory source. The (111) reflections were registered on a high-resolution CCD detector (Rigaku) with a pixel size of $2.16 \mu \mathrm{m}$. The crystal \#1 demonstrates number of defects at the corners and dislocations near the edges (Figure 2). On the microscopy image at the 
central part one can see the area with extended defects. These defects are not visible at the X-ray topography image due to small extinction depth of $\mathrm{x}$-rays for (111) reflection $-2.23 \mu \mathrm{m}$, while optical microscopy reveals all the defects throughout the plate thickness. The area at the right bottom part of the crystal is dislocation-free with the size of $1.7 \square 1.6$ $\mathrm{mm}$.

The crystal \#2 exhibit similar features with the dislocations close to the one edge of the plate, three-lobe defect at one of the corners and scratches on the surface. This crystal demonstrates larger $1.8 \square 2.3 \mathrm{~mm}$ dislocation-free area.
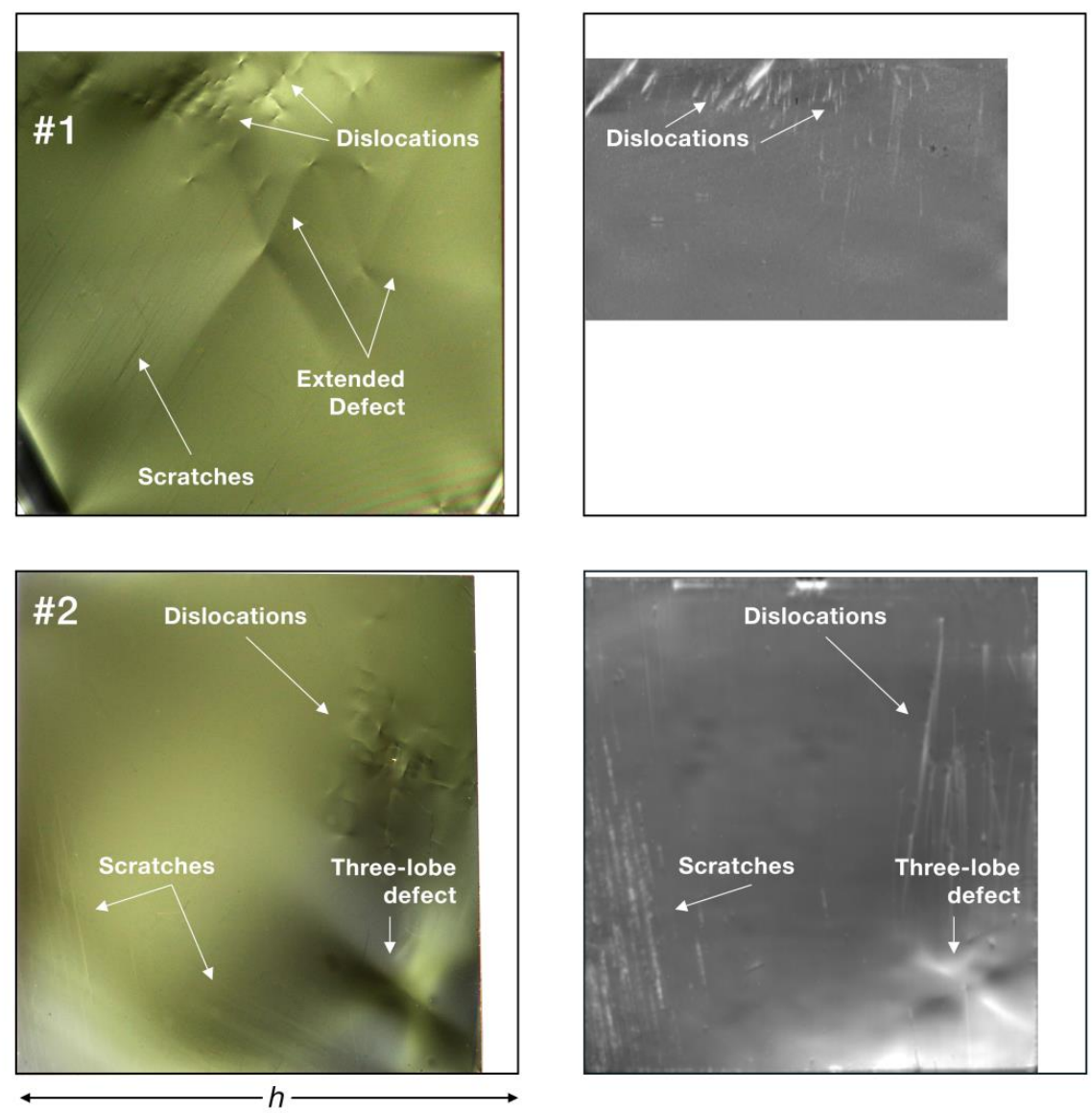

Figure 2. Optical polarization microscopy (left) and Bragg topography (right) images for (111)-oriented diamond plates ( $h$ denotes the $3.5 \mathrm{~mm}$ scale).

The integral RCs from the overall area obtained by the high resolution RCI are shown at Figure 3 together with theoretical Darwin curves. The theoretical curve for diamond (333) reflection at $14.41 \mathrm{keV}$ has FWHM of $2.13 \mu \mathrm{rad}$ for the monochromatic and collimated beam and has FWHM of $2.53 \mu \mathrm{rad}$ for the current setup considering the beam divergence and the energy dispersion. The FWHM values for studied crystals were deduced as 3.65 and $5.23 \mu \mathrm{rad}$ for \#1 and \#2, respectively. The broadening of the RCs shows a significant influence of the dislocations and strain on a crystal quality. 


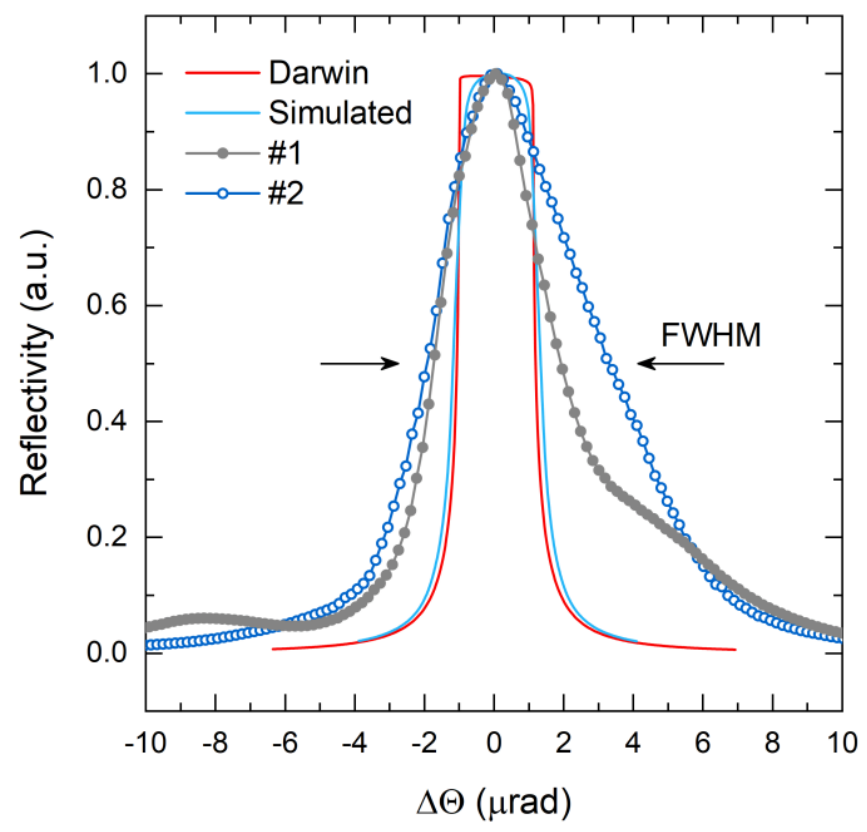

Figure 3. Integral rocking curves of the diamond crystal plates. The experimental FWHM values are much higher than the theoretical one (Darwin) and simulated curve, which considers a beam divergence and energy dispersion of the setup.

Rocking curve maps representing FWHM and COM values are shown at Figure 4. FWHM maps show the perfection of the crystal lattice and COM indicates the presence of strain in the crystal. The FWHM map of the crystal \#1 shows the values variation from 1.9 up to $5.2 \mu \mathrm{rad}$. The effect of RC narrowing is observed at the bottom left corner and around dislocations region. We should note that FWHM values in range of 3-3.5 $\mu$ rad are related to the strain induced by the polishing whether values above $3.5 \mu \mathrm{rad}$ are related to the defects and dislocations in the crystals. The COM variation in the dislocation-free area is in the margin of $1.15 \mu \mathrm{rad}$. The deviation of COM values due to the defects and dislocations reach 10 and $4 \mu \mathrm{rad}$, respectively. Comparing the RCI maps with the optical microscopy image (Figure 2) reveals the presence of extended defects close to the center of the crystal. The difference between $\mathrm{x}$-ray topography image and RCI here lies in extinction depth, which is $2.23 \mu \mathrm{m}$ for (111) reflection and $16.49 \mu \mathrm{m}$ for (333) reflection. The higher order reflection allows us to discover features related to the in-depth defects.

The FWHM distribution of the crystal \#2 is similar to the crystal \#1 with values ranging from 1.9 to $5.6 \mu \mathrm{rad}$. Excessive broadening of the RCs above $5.2 \mu \mathrm{rad}$ is attributed to the presence of the three-lobe defect. In this crystal COM distribution in the defect-free area is higher compare to the crystal \#1 lying in margin of $1.49 \mu$ rad. One should note that COM distribution shows that the surface is curved or non-uniformly strained, limiting the working area. 
(a) \#1
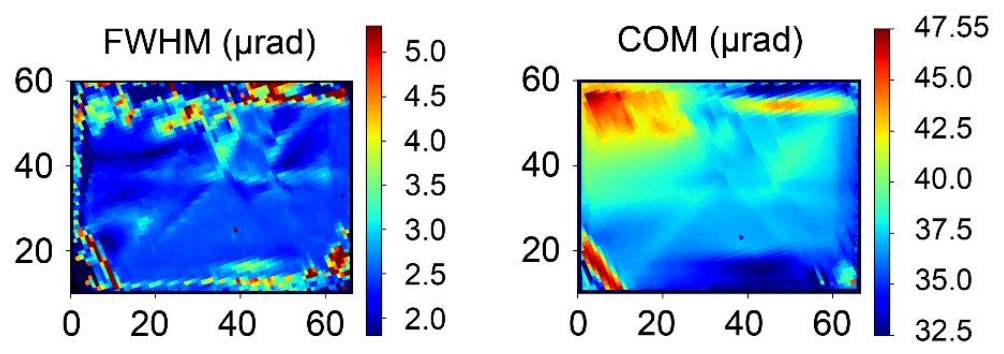

(b) \#2
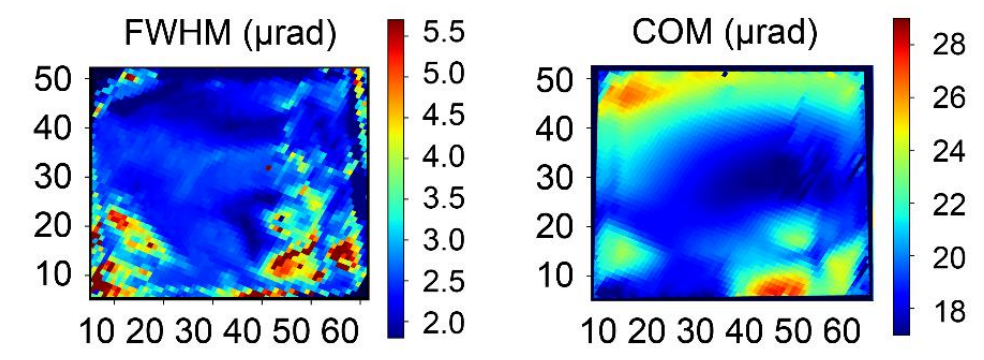

Figure 4. RCI FWHM and COM maps for crystals \#1 (a) and \#2 (b). The labels are presented in pixels, where one pixel equal to 55 $\mu \mathrm{m}$. The data outside of the plates has been removed manually.

To check the crystalline quality in detail we compare the RCs from the different parts of the diamond crystals. The points were chosen as follows: from the central part of the crystal (1), from a pronounced defect (2) and from the narrowest part (3).

The crystal \#1 demonstrates symmetric rocking curve at the Point 1 with FWHM value of $2.56 \mu$ rad, which is almost equal to the theoretical one (Figure 5a). The shape of the curve is uniform, without extra broadening, slope change and wings. The curve from the point 2 is symmetric at the bottom part and demonstrates peak splitting with FWHM of $3.57 \mu \mathrm{rad}$. This region in the crystal possess strains due to polishing. Narrowing of the rocking curve (FWHM $=1.97 \mu \mathrm{rad}$ ) from the point 3 can be described by small distortions in a crystal cell, an overall mosaicism and curved surface leading to a multiple diffraction effect [25]. The scan step of $0.17 \mu \mathrm{rad}$ should be considered.

The RC from the Point 1 of the crystal \#2 is narrow, with FWHM of $2.40 \mu \mathrm{rad}$. (Figure 5b). The result obtained from the point 3 demonstrates that defect area cannot be considered as the working area in optical applications due to its high broadening and overall complex shape including another reflection. In the narrowest part of the crystal (point 3) the FWHM value is $2.03 \mu \mathrm{rad}$, similar to that in the crystal \#1. One can note that COM values shifts by up to $4 \mu \mathrm{rad}$, demonstrating strains or crystal curvature. 

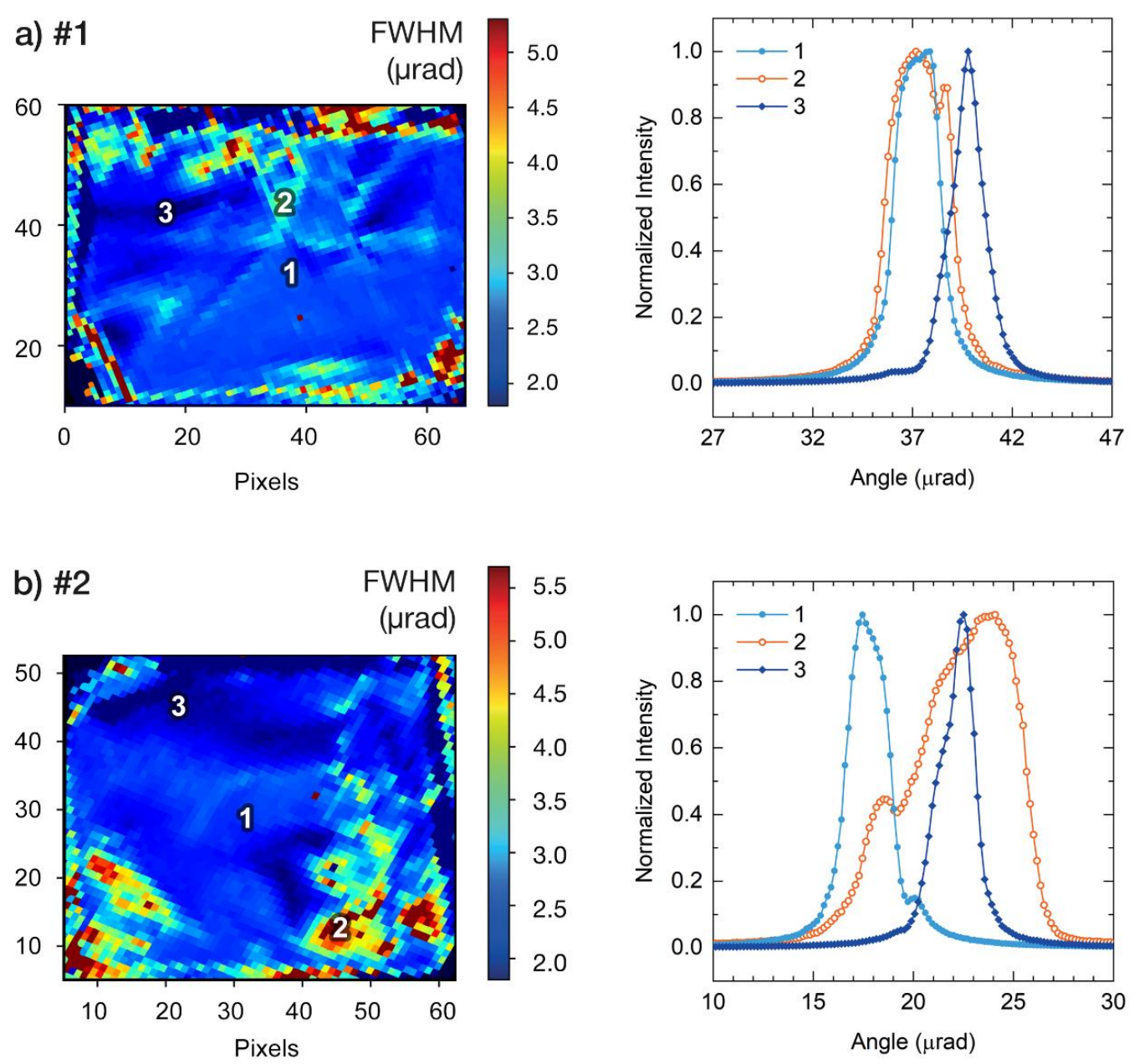

Figure 5. FWHM maps and rocking curves corresponding to the indicated pixels in comparison with integral RC for diamond plates for crystal \#1 (a) and \#2 (b). The pixels were taken from the central area (1), defect (2) and the lowest FWHM value (3).

For more precise estimation of a working area in the diamond crystals we reduced the range of values at FWHM maps. The areas with dislocations and significant strain represented by values higher than $4.0 \mu \mathrm{rad}$ were cut. The lowermost values were limited to $1.9 \mu \mathrm{rad}$. Considering this we plotted FWHM maps and made a statistical distribution of FWHM values (Figure 6). The RMS values and standard deviation were derived as $2.60 \pm 0.38 \mu \mathrm{rad}$ and $2.64 \pm 0.45$ $\mu \mathrm{rad}$ for crystal \#1 and \#2, respectively. Working areas with deviation of $0.5 \mu \mathrm{rad}$ from the theoretical value were estimated at $1 \times 2.2$ and $1 \times 1.6 \mathrm{~mm}$ for crystal \#1 and \#2, respectively. Extending the deviation value to $1 \mu \mathrm{rad}$ for less demanding optical applications we received the working areas of $1.5 \times 2.8 \mathrm{~mm}(\# 1)$ and $1.4 \times 1.7 \mathrm{~mm}(\# 2)$. 

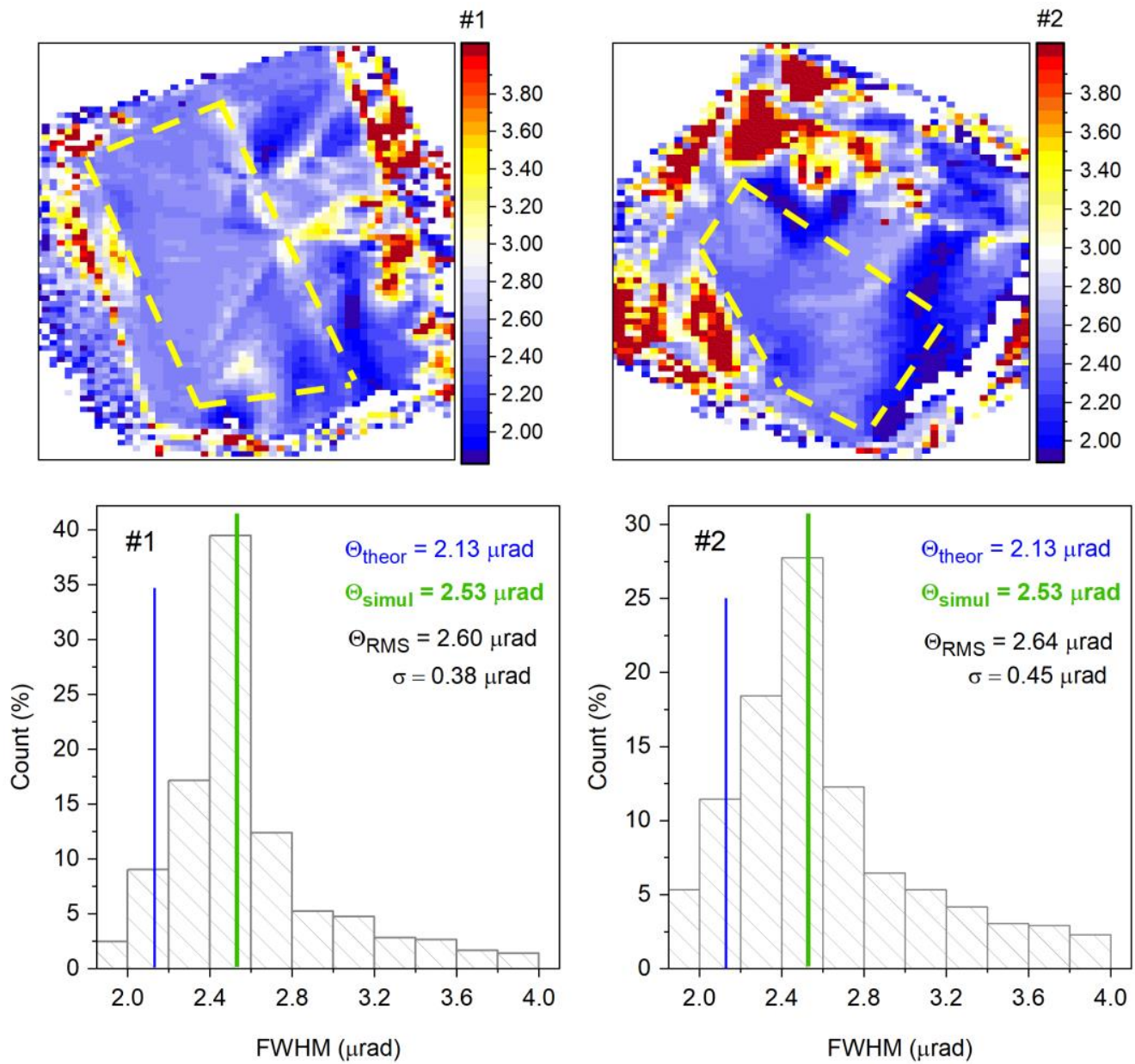

Figure 6. FWHM maps and distribution histograms of diamond plates artificially limited from 1.9 to $4.0 \mu$ rad. The data responsible for defects and highly strained regions due to crystal cut and polishing were removed. Yellow rectangles on FWHM maps indicate regions that fall to the scope of $1 \mu \mathrm{rad}$ deviation from the theoretical value. Solid blue lines show FWHM of the Darwin and simulated curves.

As a result, the crystal \#1 has larger working area and lower FWHM mean value and deviation comparing to the crystal \#2 making it more suitable for optical applications. The region around working area can be used for crystal mounting. The crystal \#2 has threefold defect, which takes around $30 \%$ of the surface. The dislocations visible at the $10 \%$ of $I_{\max }$ map take another $10 \%$ near the defect. The miscut angle of the crystal \#1 of 6.06 degrees altogether with internal strains and crystal curvature give COM shift up to $4 \mu \mathrm{rad}$. However, the standard deviation of COM, $\delta \theta_{\text {Сом }}$, is $1.15 \mu \mathrm{rad}$, and does not exceed $1.6 \mu \mathrm{rad}$ in the working area. The situation with the crystal \#2 is more critical with the standard deviation of $1.49 \mu \mathrm{rad}$ and the shift up to $1.9 \mu \mathrm{rad}$ in the working area making it less suitable for optical applications (Figure 7). To avoid aforementioned features, one need to improve the cutting process to receive a miscut angle lower than 2 degrees and polishing process to reduce the curvature and additional strains. This will allow to achieve at least $2.5 \times 2.5 \mathrm{~mm}^{2}$ working area enough for XFEL and $4^{\text {th }}$-generation sources optical applications. Considering the paper of Khokhryakov, where the size of the plate was as high as $6.9 \times 3.7 \mathrm{~mm}^{2}$ one can receive even larger working area [21]. 

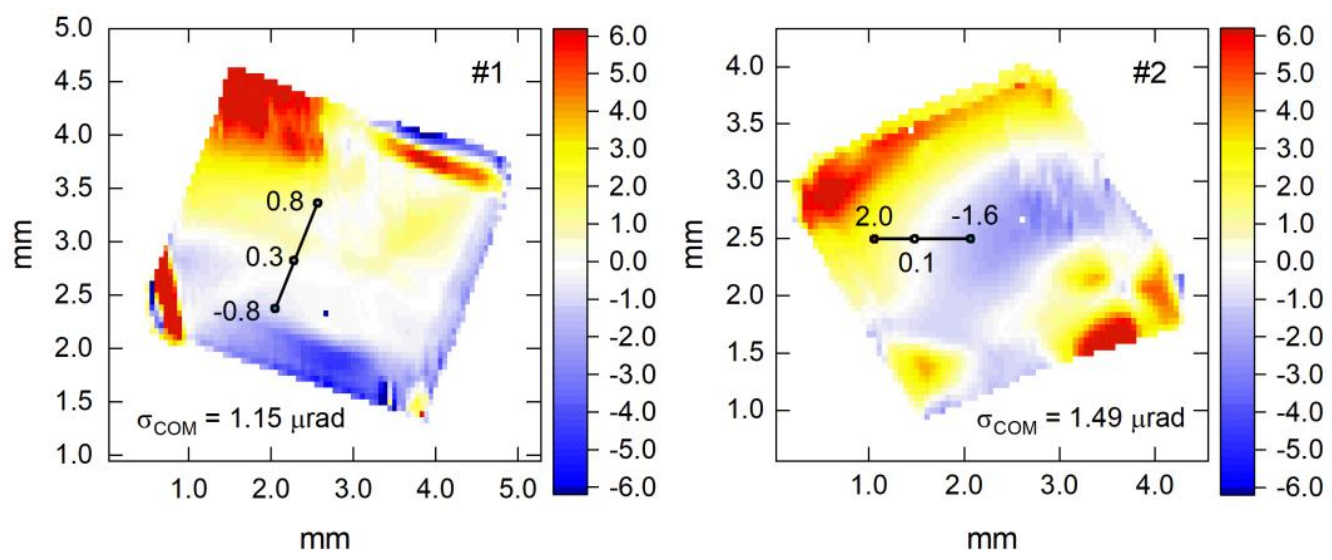

Figure 7. COM maps of diamond plates plotted from -5.5 to $5.5 \mu \mathrm{rad}$. The line profiles show COM distribution example in the working area. The colorbar shows values in $\mu \mathrm{rad}$

To confirm the applicability of the marked in Figure 6 working areas we have made integral RCs and compare them with the simulated curve (Figure 8). An overall shape of the integral RCs is uniform, without secondary peaks or broad wings at the edges. The FWHM values are $2.93 \mu \mathrm{rad}(\# 1)$ and $3.33 \mu \mathrm{rad}(\# 2)$, exceeding experimentally calculated value of $2.53 \mu \mathrm{rad}$ by 0.4 and $0.8 \mu \mathrm{rad}$ for crystal \#1 and \#2, respectively. The crystal \#1 possess better quality in comparison with the crystal \#2. It has larger working area and FWHM values close to the simulated and smaller deviation. The integral RC, which also considers COM distribution, demonstrates the broadening value of $0.4 \mu \mathrm{rad}$ making the crystal suitable for almost all optical applications.

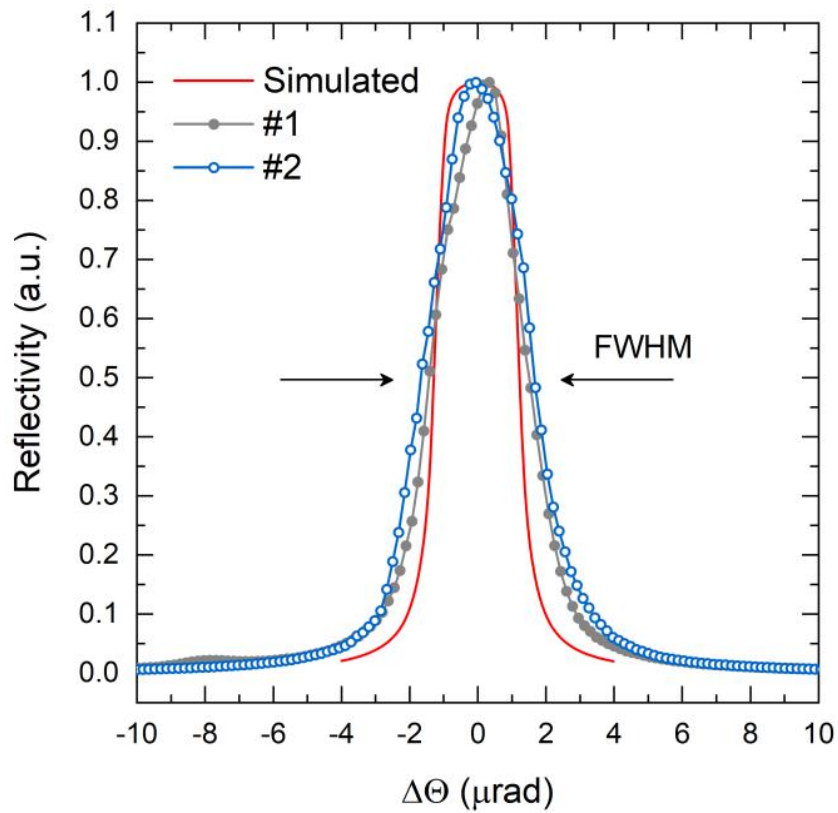

Figure 8. Integral rocking curves taken from the dislocation-free areas indicated at Figure 6 showing that the deviation of FWHM falls in the scope of $0.8 \mu \mathrm{rad}$

Finally, we preformed RCI of nitrogen free (100) oriented diamond plate (\#3) with around $2 \mathrm{~mm}^{2}$ surface area. The FWHM and COM maps are shown at Figure 9. A (400) reflection of diamond have been chosen for RCI measurements with theoretical FWHM of $4.1 \mu \mathrm{rad}$ at $14.41 \mathrm{keV}$. The FWHM map shows the distribution from 4.0 to 5.0 $\mu \mathrm{rad}$ with the mean value of $4.15 \mu \mathrm{rad}$ and the standard deviation of $0.23 \mu \mathrm{rad}$. It should be noted that the crystal \#3 is mostly defect free, but contains insignificant strains close to the edges. The COM distribution indicates small bending of the crystal with the deviation up to $0.9 \mu \mathrm{rad}$. The working area was estimated as $1.0 \times 1.5 \mathrm{~mm}$ or $65 \%$ of the whole crystal surface. However, all rocking curves contain secondary diffraction peak shifted by $10 \mu \mathrm{rad}$. It could be related to the 
strong atomic planes tilt - mosaic domains, resulting at additional peaks of the same reflection order close to the primary one [26]. In addition, the tilt and strain influence the FWHM and COM of a rocking curves and could be separated by measuring RCs at $180^{\circ}$ azimuthal rotation around the reciprocal lattice vector for the X-ray diffraction [19].

All the parameters were gathered in Table 1.
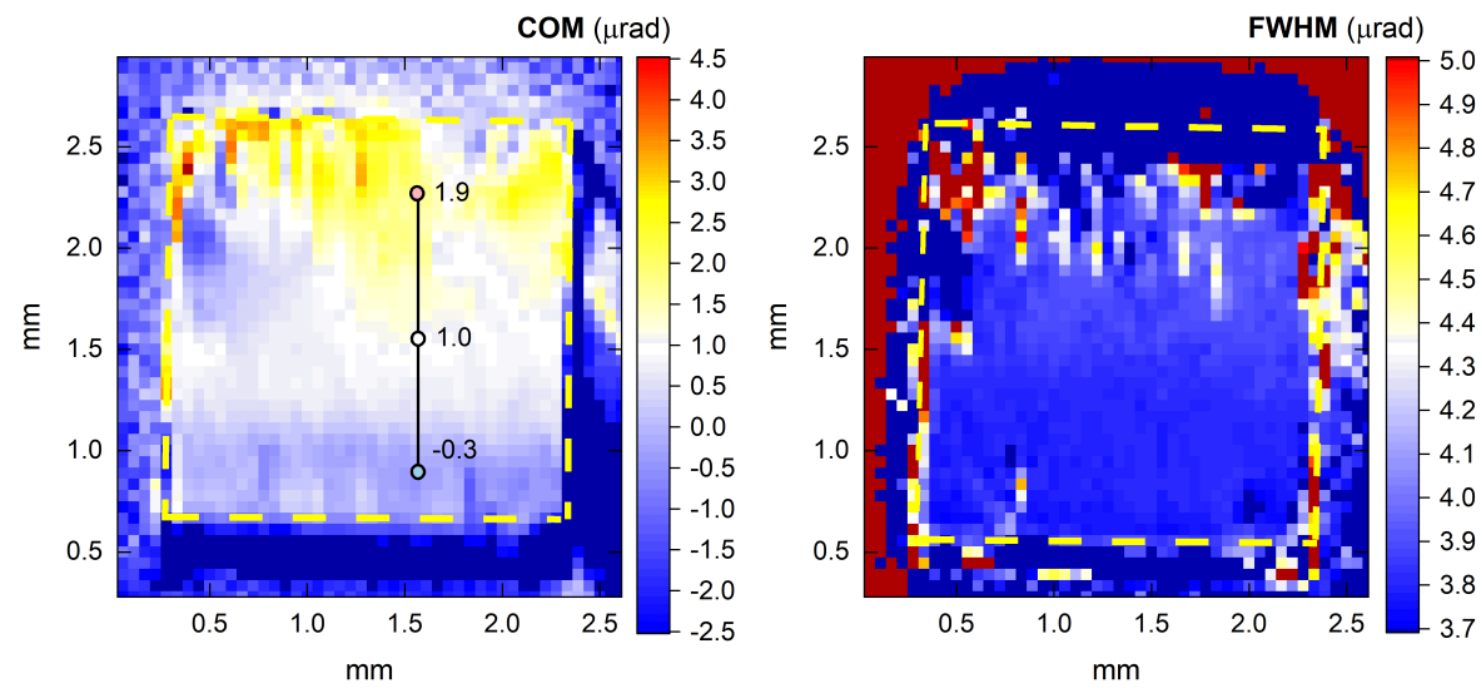

Figure 9. COM (left image) and FWHM (right image) of (100) diamond plate of IIa type (\#3). Yellow rectangle indicates the crystal area and the line profile shows center of mass distribution across the crystal

Table 1. Size and characteristics of the diamond plates derived from the RCI. $\theta$ and $\delta \theta_{\text {FWHM }}$ are the FWHM values and standard

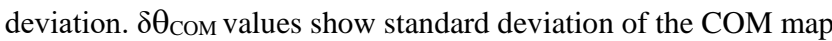

\begin{tabular}{|l|l|l|l|l|l|l|l|l|}
\hline$\#$ & $\begin{array}{l}\text { Size, } \\
\mathrm{mm}\end{array}$ & $\begin{array}{l}\text { Miscut } \\
\text { angle, }\end{array}$ & $\begin{array}{l}\theta_{\mathrm{RMS},} \\
\mu \mathrm{rad}\end{array}$ & $\begin{array}{l}\delta \theta_{\text {FWHM }}, \\
\mu \mathrm{rad}\end{array}$ & $\begin{array}{l}\delta \theta_{\mathrm{COM}}, \\
\mu \mathrm{rad}\end{array}$ & $\begin{array}{l}\text { Working area, } \\
\mathrm{mm}\end{array}$ & $\begin{array}{l}\text { Working area } \\
\text { integral FWHM, } \\
\mu \mathrm{rad}\end{array}$ & $\begin{array}{l}\text { Working } \\
\theta_{\mathrm{FWHM}} \leq 3 \\
\mathrm{~mm}\end{array}$ \\
\hline $\mathbf{1}$ & $3.4 \times 3.2$ & 3.67 & 2.60 & 0.38 & 1.15 & $1.0 \times 2.2$ & 2.93 & $1.5 \times 2.8$ \\
\hline $\mathbf{2}$ & $2.9 \times 3.1$ & 6.06 & 2.64 & 0.45 & 1.49 & $1.0 \times 1.6$ & 3.33 & $1.4 \times 1.7$ \\
\hline $\mathbf{3}$ & $2.1 \times 2.0$ & 0.10 & 4.15 & 0.23 & 1.5 & $1.0 \times 1.5$ & 4.31 & $1.8 \times 1.6$ \\
\hline
\end{tabular}

The last, but not least is the wavefront preserving criterion, critical for the new synchrotron sources and XFELs optics. An angular mapping in RCI provides quantitative characterization of the wavefront of the reflected radiation. By estimation of the COM deviation across the crystal one can compare it with the angular divergence of the incident X-ray beam, $\Delta \psi$, provided by the light source [17]:

$$
\delta \theta_{\text {COM }} \ll \Delta \psi
$$

A typical angular divergence of an undulator-based beamline of a $3^{\text {rd }}$ generation synchrotron is $5 \mu$ rad, so in our case of COM deviation not exceeding $1.5 \mu \mathrm{rad}$ this criterion is satisfied. However, for XFEL sources, like LCLS or Eu-XFEL with the angular divergence around $1 \mu \mathrm{rad}$, the currently studied diamond plates are unsuitable.

For the thorough characterization of the wavefront an additional experiment using Talbot effect is required. A phase-shift grating [27-30] or multilens interferometer [31] are needed to characterize the X-ray beam properties and its change due to optics elements. The experiment is similar for gratings and interferometers and described in detail by Rack [27]. The Talbot effect is used to access the coherence properties of the reflected beam as well as long propagation distance imaging to study the beam homogeneity. A high-resolution imaging detector translated at short propagation distances along the beam is used to measure the visibility of a diffraction grating in transmission geometry placed close to the studied crystal. The change of the fringe visibility as a function of distance between the grating and the detector gives access to the angular source size, while detector at longer propagation distances allows to measure the 
homogeneity of the beam. Its noteworthy to keep in mind the quality of the gratings and interferometers using for measurements that by itself must not affect an X-ray beam.

\section{CONCLUSIONS}

In summary we showed that $\mathrm{Ib}$ (111)-oriented diamond plates can possess a high diffraction quality despite the nitrogen presence even in comparison to the IIa diamond plate prepared by the same technique. Three diamond crystals with thickness of 200 micrometers and 2-3 $\mathrm{mm}^{2}$ area were studied by high-resolution RCI. The results demonstrate the FWHM mean value at the dislocation- and strainfree areas lies within $0.5 \mu \mathrm{rad}$ deviation from the theoretical value that is suitable for XFEL and 4th generation synchrotron sources optical applications like beam splitters and monochromators. The working area of the crystal \#1 and \#3 exceeds 50\% of the whole crystal surface. Further improvement of the cutting and polishing processes will lead to the large diamond crystals (more than $\left.6 \mathrm{~mm}^{2}\right)$ with larger working area.

\section{ACKNOWLEDGMENTS}

The authors thank I.N. Kupriyanov for FTIR characterization of diamond plates. This research was funded by RFBR, project number 19-29-12040, and the Ministry of Science and Higher Education of the Russian Foundation on state assignment of IGM SB RAS.

\section{REFERENCES}

[1] D. Einfeld, J. Schaper, M. Plesko, Design of a diffraction limited light source (DIFL), in: Proc. Part. Accel. Conf., IEEE, 1995: pp. 177-179. https://doi.org/10.1109/PAC.1995.504602.

[2] I. Sergueev, R. Döhrmann, J. Horbach, J. Heuer, Angular vibrations of cryogenically cooled double-crystal monochromators, J. Synchrotron Radiat. 23 (2016) 1097-1103. https://doi.org/10.1107/S1600577516011188.

[3] P. Theveneau, R. Baker, R. Barrett, A. Beteva, M.W. Bowler, P. Carpentier, H. Caserotto, D. De Sanctis, F. Dobias, D. Flot, M. Guijarro, T. Giraud, M. Lentini, G.A. Leonard, M. Mattenet, A.A. McCarthy, S.M. McSweeney, C. Morawe, M. Nanao, D. Nurizzo, S. Ohlsson, P. Pernot, A.N. Popov, A. Round, A. Royant, W. Schmid, A. Snigirev, J. Surr, C. Mueller-Dieckmann, The upgrade programme for the structural biology beamlines at the European synchrotron Radiation Facility-High throughput sample evaluation and automation, J. Phys. Conf. Ser. 425 (2013). https://doi.org/10.1088/1742-6596/425/1/012001.

[4] M. Polikarpov, I. Snigireva, J. Morse, V. Yunkin, S. Kuznetsov, A. Snigirev, Large-acceptance diamond planar refractive lenses manufactured by laser cutting, J. Synchrotron Radiat. 22 (2015) 23-28. https://doi.org/10.1107/S1600577514021742.

[5] S. Terentyev, V. Blank, S. Polyakov, S. Zholudev, A. Snigirev, M. Polikarpov, T. Kolodziej, J. Qian, H. Zhou, Y. Shvyd'Ko, Parabolic single-crystal diamond lenses for coherent X-ray imaging, Appl. Phys. Lett. 107 (2015) 1-6. https://doi.org/10.1063/1.4931357.

[6] P. Medvedskaya, I. Lyatun, S. Shevyrtalov, M. Polikarpov, I. Snigireva, V. Yunkin, A. Snigirev, Diamond refractive micro-lenses for full-field X-ray imaging and microscopy produced with ion beam lithography, Opt. Express. 28 (2020). https://doi.org/10.1364/OE.384647.

[7] K.-J. Kim, L. Assoufid, F.-J. Decker, Z. Huang, R. Lindberg, G. Marcus, T. Raubenheimer, X. Shi, D. Shu, Y. Shvyd'ko, M. White, and D. Zhu, "Test of an X-ray cavity using double-bunches from the lcls cu-linac," Tech. rep., SLAC National Accelerator Lab., Menlo Park, CA (United States) (2019).

[8] W. Hua, G. Zhou, Z. Hu, S. Yang, K. Liao, P. Zhou, X. Dong, Y. Wang, F. Bian and J. Wang, "On-line monitoring of the spatial properties of hard X-ray free-electron lasers based on a grating splitter", J. Synchrotron Rad. 26, 619-628 (2019). https://doi.org/10.1107/S1600577519001681

[9] G. Grübel, D. Abernathy, G. Vignaud, M. Sanchez Del Rio, A. Freund, A diamond double-crystal transmission 
monochromator for the TROIKA II station at ESRF, Rev. Sci. Instrum. 67 (1996) 3349.

https://doi.org/10.1063/1.1147490.

[10] M. Yabashi, S. Goto, Y. Shimizu, K. Tamasaku, H. Yamazaki, Y. Yoda, M. Suzuki, Y. Ohishi, M. Yamamoto, T. Ishikawa, Diamond double-crystal monochromator for SPring-8 undulator beamlines, AIP Conf. Proc. 879 (2007) 922-925. https://doi.org/10.1063/1.2436212.

[11] H. Sumiya, S. Satoh, High-pressure synthesis of high-purity diamond crystal, Diam. Relat. Mater. 5 (1996) 1359-1365. https://doi.org/10.1016/0925-9635(96)00559-6.

[12] Q. Han, B. Liu, M. Hu, Z. Li, X. Jia, M.-Z. Li, H. Ma, S.-S. Li, H. Xiao, Y. Li, Design an Effective Solution for Commercial Production and Scientific Research on Gem-Quality, Large, Single-Crystal Diamond by High Pressure and High Temperature, Cryst. Growth Des. 11 (2011) 1000-1005. https://doi.org/10.1021/cg100940b.

[13] S.N. Polyakov, V.N. Denisov, N. V Kuzmin, M.S. Kuznetsov, S.Y. Martyushov, S.A. Nosukhin, S.A. Terentiev, V.D. Blank, Diamond \& Related Materials Characterization of top-quality type IIa synthetic diamonds for new X-ray optics 论㑔, Diam. Relat. Mater. 20 (2011) 726-728. https://doi.org/10.1016/j.diamond.2011.03.012.

[14] Y.N. Palyanov, I.N. Kupriyanov, A.F. Khokhryakov, V.G. Ralchenko, Crystal Growth of Diamond, Second Edi, Elsevier B.V., 2015. https://doi.org/10.1016/B978-0-444-63303-3.00017-1.

[15] R.C. Burns, A.I. Chumakov, S.H. Connell, D. Dube, HPHT growth and x-ray characterization of high-quality type IIa diamond, 364224 (2009). https://doi.org/10.1088/0953-8984/21/36/364224.

[16] S. Stoupin, Novel diamond X-ray crystal optics for synchrotrons and X-ray free-electron lasers, Diam. Relat. Mater. 49 (2014) 39-47. https://doi.org/10.1016/j.diamond.2014.08.002.

[17] S. Stoupin, S. Antipov, J.E. Butler, A. V. Kolyadin, A. Katrusha, Large-surface-area diamond (111) crystal plates for applications in high-heat-load wavefront-preserving X-ray crystal optics, J. Synchrotron Radiat. 23 (2016) 1118-1123. https://doi.org/10.1107/S1600577516011796.

[18] Y. Shvyd'Ko, V. Blank, S. Terentyev, Diamond X-ray optics: Transparent, resilient, high-resolution, and wavefront preserving, MRS Bull. 42 (2017) 437-444. https://doi.org/10.1557/mrs.2017.119.

[19] A.T. MacRander, S. Krasnicki, Y. Zhong, J. Maj, Y.S. Chu, Strain mapping with parts-per-million resolution in synthetic type-Ib diamond plates, Appl. Phys. Lett. 87 (2005) 1-3. https://doi.org/10.1063/1.2126790.

[20] J. Hoszowska, A.K. Freund, E. Boller, J.P.F. Sellschop, G. Level, J. Härtwig, R.C. Burns, M. Rebak, J. Baruchel, Characterization of synthetic diamond crystals by spatially resolved rocking curve measurements, J. Phys. D. Appl. Phys. 34 (2001) A47-A51. https://doi.org/10.1088/0022-3727/34/10a/311.

[21] A.F. Khokhryakov, Y.N. Palyanov, I.N. Kupriyanov, Y.M. Borzdov, A.G. Sokol, J. Härtwig, F. Masiello, Crystal growth and perfection of large octahedral synthetic diamonds, J. Cryst. Growth. 317 (2011) 32-38. https://doi.org/10.1016/j.jcrysgro.2011.01.011.

[22] Y.N. Palyanov, Y.M. Borzdov, A.F. Khokhryakov, I.N. Kupriyanov, A.G. Sokol, Effect of nitrogen impurity on diamond crystal growth processes, Cryst. Growth Des. 10 (2010) 3169-3175. https://doi.org/10.1021/cg100322p.

[23] A.M. Zaitsev, Optical Properties of Diamond, Springer Berlin Heidelberg, Berlin, Heidelberg, 2001. https://doi.org/10.1007/978-3-662-04548-0.

[24] H.C. Wille, H. Franz, R. Röhlsberger, A. Caliebe, F.U. Dill, Nuclear resonant scattering at PETRA III : BBBBrillant opportunities for nano - And extreme condition science, J. Phys. Conf. Ser. 217 (2010). https://doi.org/10.1088/1742-6596/217/1/012008.

[25] S.L. Chang, Solution to the X-ray Phase Problem Using Multiple Diffraction - a Review, 1987. https://doi.org/10.1080/08893118708075481.

[26] B.K. Tanner, P.J. Parbrook, B. Lunn, J.H.C. Hogg, A.M. Keir, A.D. Johnson, Observation of hardening during relaxation of InGaAs on GaAs, J. Phys. D. Appl. Phys. 36 (2003) A198-A201. https://doi.org/10.1088/00223727/36/10A/340.

[27] A. Rack, T. Weitkamp, L. Assoufid, T. Rack, I. Zanette, C. Morawe, R. Kluender, C. David, Protocol to study wavefront preservation capabilities of reflective X-ray optics with coherent synchrotron light, Nucl. Instruments Methods Phys. Res. Sect. A Accel. Spectrometers, Detect. Assoc. Equip. 710 (2013) 101-105. https://doi.org/10.1016/j.nima.2012.10.116.

[28] K. Li, Y. Liu, M. Seaberg, M. Chollet, T.M. Weiss, A. Sakdinawat, Wavefront preserving and high efficiency diamond grating beam splitter for X-ray free electron laser, Opt. Express. 28 (2020) 10939. https://doi.org/10.1364/oe.380534.

[29] Y. Liu, M. Seaberg, D. Zhu, J. Krzywinski, F. Seiboth, C. Hardin, D. Cocco, A. Aquila, B. Nagler, H.J. Lee, S. Boutet, Y. Feng, Y. Ding, G. Marcus, A. Sakdinawat, High-accuracy wavefront sensing for X-ray free electron 
lasers, Optica. 5 (2018) 967. https://doi.org/10.1364/OPTICA.5.000967.

[30] S. Rutishauser, L. Samoylova, J. Krzywinski, O. Bunk, J. Grünert, H. Sinn, M. Cammarata, D.M. Fritz, C. David, Exploring the wavefront of hard X-ray free-electron laser radiation, Nat. Commun. 3 (2012) 947. https://doi.org/10.1038/ncomms1950.

[31] A. Snigirev, I. Snigireva, M. Lyubomirskiy, V. Kohn, V. Yunkin, S. Kuznetsov, X-ray multilens interferometer based on Si refractive lenses, Opt. Express. 22 (2014) 25842. https://doi.org/10.1364/OE.22.025842. 